\title{
Chopstick Injury: Successful Stent-Graft Therapy for Traumatic Left Subclavian Artery Aneurysm
}

\author{
Tatsuo Ueda ${ }^{1}$, Hiroyuki Tajima ${ }^{2}$, Satoru Murata ${ }^{1}$, \\ Ryo Takagi ${ }^{1}$, Hiroyuki Yokota ${ }^{3}$ and Shin-ichiro Kumita ${ }^{1}$ \\ ${ }^{1}$ Department of Radiology, Nippon Medical School Hospital, Tokyo, Japan \\ ${ }^{2}$ Department of Center for Minimally Invasive Treatment, Nippon Medical School Musashi Kosugi Hospital, Kanagawa, Japan \\ ${ }^{3}$ Department of Emergency and Critical Care Medicine, Nippon Medical School Hospital, Tokyo, Japan
}

\begin{abstract}
Traumatic chopstick injury is very rare, especially in the vascular system. We present an unusual case of a 19-year-old man who presented at the emergency department after being stabbed with a chopstick by his elder brother. Computed tomography revealed a left subclavian artery pseudoaneurysm, which increased from $4 \mathrm{~mm}$ to $7 \mathrm{~mm}$ in diameter within 47 days. We successfully deployed a Niti-S stent graft for the increasing aneurysm, thereby avoiding a surgical operation. Balloon angioplasty was added for re-stenosis 8 months after the first intervention. (J Nippon Med Sch 2017; 84: 41-44)
\end{abstract}

Key words: chopstick, left subclavian artery, pseudoaneurysm, stent graft

\section{Introduction}

Traumatic chopstick injury rarely induces subclavian artery pseudoaneurysm, which is potentially life threatening because of the risk of rupture, distal embolization, and thrombosis. Open surgical repair is the standard treatment modality for subclavian artery aneurysms, but their location in the intrathoracic segment necessitates an invasive approach with sternotomy or lateral thoracotomy ${ }^{1}$. Recently, a less invasive endovascular treatment using stent grafts has been reported as a viable alternative to open repair. Endovascular repair offers an attractive alternative to open surgery for the management of injuries, and its safety and efficacy has been previously reported ${ }^{2}$.

Here, we report a traumatic left subclavian artery aneurysm induced by chopstick insertion, which was successfully treated with a stent graft.

\section{Case Report}

A 19-year-old man presented at the emergency department with a chopstick stuck in his left neck after a fight with his older brother. His blood pressure was 112/60 $\mathrm{mmHg}$ and pulse 96 beats/min. Thoracic contrastenhanced computed tomography (CT) revealed a left subclavian artery aneurysm (Fig. 1a) and massive right hemopneumothorax, which was drained by a thoracic tube. There was no remarkable past history or family history. As the patient's hemodynamic state was stable, conservative treatment was chosen, and he was discharged 13 days after the event. Follow-up CT 47 days after the onset revealed marked enlargement of the aneurysm (Fig. 1b), and we decided to treat it by endovascular intervention. The left vertebral artery diverged directly from the thoracic aorta. Under local anesthesia, the right femoral artery was punctured, and a 9-Fr sheath (Medikit Co., Tokyo, Japan) was inserted. Thoracic and left subclavian angiogram showed that the aneurysm was located $4 \mathrm{~cm}$ distal to the origin of the left subclavian artery and was $7 \mathrm{~mm}$ in size with a 5 -mm-wide neck (Fig. 2a). A 0.035-inch Radifocus guidewire (Terumo, Tokyo, Japan) was then advanced to the distal side of the aneurysm, and a 5-Fr headhunter type catheter (Medikit Co., Tokyo, Japan) was inserted to the distal left subclavian artery. Over a 0.035-inch exchange-length stiff wire (Amplatz Extra-Stiff Wire Guides; Cook Medical, Bloomington, IN, USA), a Niti-S ComVi stent graft (Century Medical, Tokyo, Japan) of 8-mm width and 60-mm length was advanced to the aneurysm and then released. After

Correspondence to Tatsuo Ueda, MD, PhD, Department of Radiology, Nippon Medical School Hospital, 1-1-5 Sendagi, Bunkyo-

ku, Tokyo 113-8603, Japan

E-mail: s9015@nms.ac.jp

Journal Website (http://www.nms.ac.jp/jnms/) 

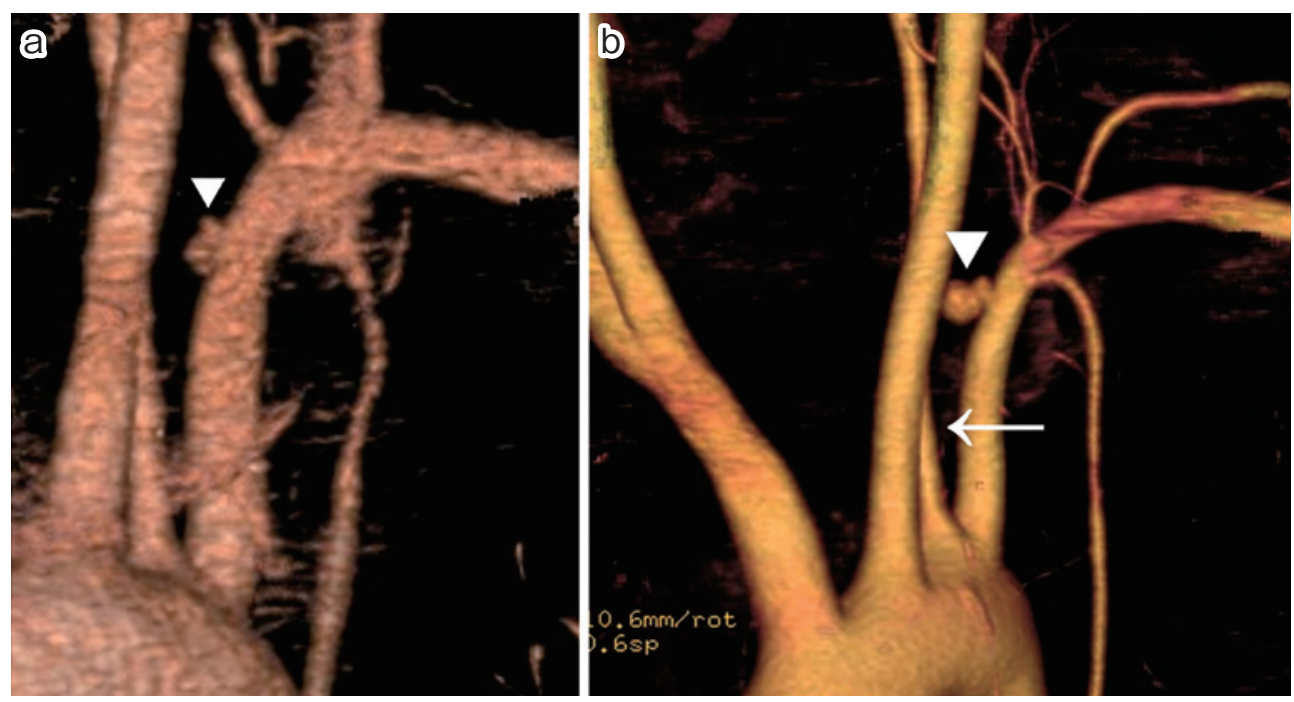

Fig. 1 Three-dimensional computed tomography (day 0 and day 47)

Three-dimensional computed tomography shows a saccular aneurysm located $4 \mathrm{~cm}$ distal to the origin of the left subclavian artery and $4 \mathrm{~mm}$ in diameter (a: arrowhead).

The aneurysm increases rapidly in diameter to $7 \mathrm{~mm}$ on day 47 (b: arrowhead). Left vertebral artery is diverging directly from the thoracic aorta (b: arrow).
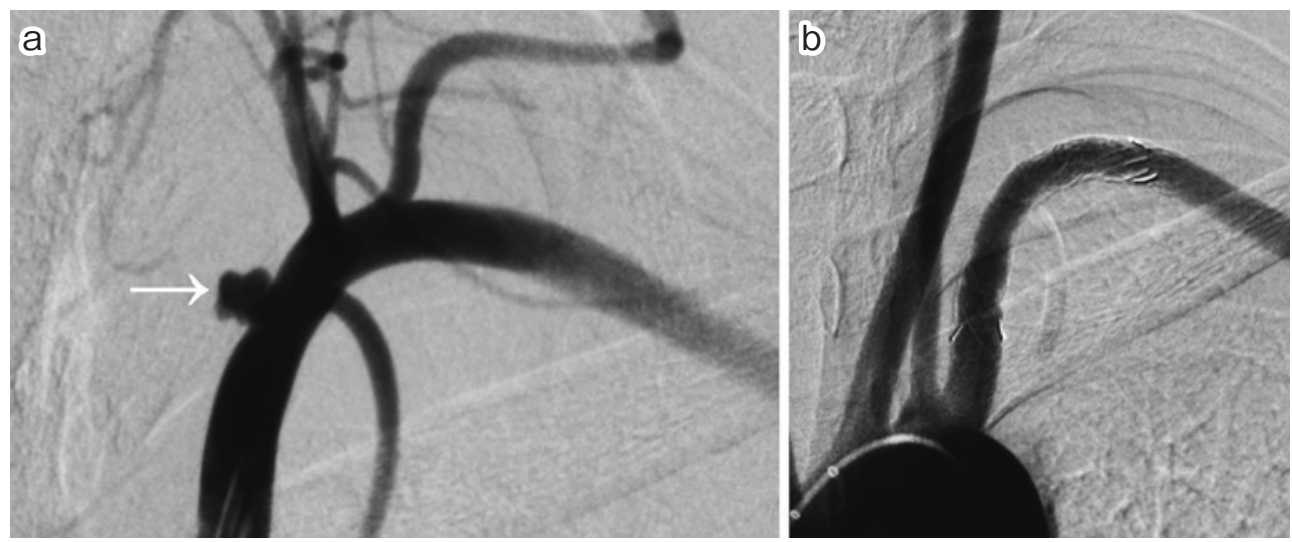

Fig. 2 Arteriogram (day 54)

Left subclavian angiogram shows saccular aneurysm with 5-mm-wide neck (a).

After deployment of a stent graft (Niti-S ComVi $8 \mathrm{~mm} \times 60 \mathrm{~mm}$ ) and fitting with $6 \mathrm{~mm}$ and $7 \mathrm{~mm}$ balloons, angiogram shows no endoleak (b).

manual inflation of a 7-mm balloon catheter (Power Flex P3, Cordis, Fremont, CA, USA), no endoleaks remained (Fig. 2b). No technical complication was seen during or after the procedure, and the patient was discharged 13 days after admission.

We prescribed two oral antiplatelet therapies (aspirin $100 \mathrm{mg}$ /day, cilostazol $200 \mathrm{mg}$ /day) to the patient after the procedure, which was continued during the followup. A follow-up CT 11 days after the procedure indicated no aneurysm of the left subclavian artery and no stentrelated complications. However, the patient developed mild stenosis with slight thrombus on CT 8 months after treatment, which was successfully treated with a second angioplasty (Fig. 3). We continued the oral antiplatelet therapies with the same dosage of drugs after the second intervention. Unfortunately, the patient was subsequently lost to follow-up because he died of suicide.

The patient provided informed consent for the treatment performed; due to the nature of the procedure, local ethics committee approval was not required.

\section{Discussion}

Penetrating injuries with chopsticks are peculiar accidents in Asian culture. There have been some reports of 

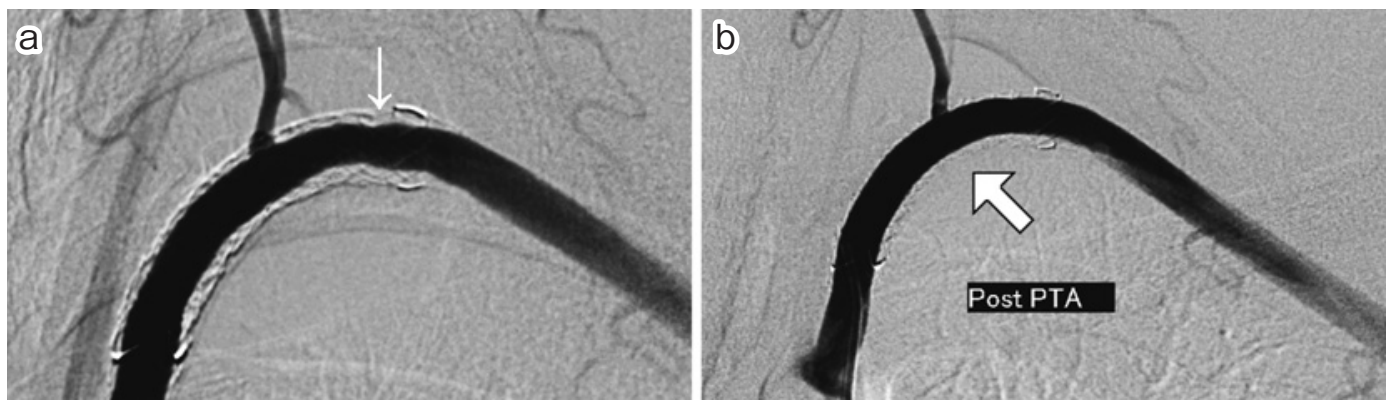

Fig. 3 Angiogram

Angiogram illustrates mild neo-intimal ingrowth (a, arrow).

There remains no stenosis in the stent graft after balloon angioplasty (b).

penetrating craniofacial injury due to chopsticks ${ }^{3}$. Such injuries are characteristically incidental, and involve simple penetrations without multiple stabs or migrations in most cases. The mechanism of simple penetrating trauma due to chopsticks has been claimed to result in less vascular injury compared to trauma due to other instruments such as knife blades or other sharp objects ${ }^{3}$. Therefore, cases of penetrating vascular injury by chopsticks are extremely rare.

Aneurysms of the subclavian artery are infrequently seen peripheral aneurysms. There are two types of aneurysms, true aneurysms and pseudoaneurysms. Most pseudoaneurysms are caused by trauma ${ }^{4}$, and the most traumatic pseudoaneurysms are caused by a gunshot or catheterization $^{5}$. In our case, the cause of the aneurysm was penetrating chopstick injury. To the best of our knowledge, there have been no previous reports of this occurrence.

Most true aneurysms are asymptomatic and found incidentally ${ }^{4}$. The common complications of subclavian artery aneurysm include compression, distal embolization by thrombosis, and hemorrhage by rupture, but they also have an associated risk of catastrophic brain embolization and stroke ${ }^{1}$. Therefore, even if a case is asymptomatic, it should be treated in the event that the aneurysm size increases.

Traditionally, surgical operation was the standard treatment for aneurysm. However, in 1991, Becker et al. reported the first use of a subclavian stent graft for the treatment of subclavian artery injury ${ }^{6}$. Since then, endovascular treatment of the subclavian artery with a variety of devices and approaches has been reported ${ }^{7}$. Endovascular treatment can be divided into two types, stent graft and coil embolization. The choice of management strategy depends on the location, shape, and characteristics of the aneurysm. In the case of relatively large diameter vessels like the subclavian artery, stent graft therapy is preferable because of the need to protect peripheral vessels. It is possible to use a stent graft in cases of aneurysms that are present at a distance from the vertebral artery. From the perspective of aneurysm shapes, sacculartype aneurysms with a narrow neck can be treated with coil embolization. Conversely, wide-neck aneurysms are difficult to treat with coil embolization due to the risk of coil migration and compaction. Furthermore, a stent graft should be chosen for inflammatory aneurysms or pseudoaneurysms, because if they are embolized by coil, they can easily rupture owing to fragility. In our case, we chose stent graft therapy because it was less invasive, there was no risk of vertebral artery occlusion, and the aneurysm was not saccular with a narrow neck. As an alternative method, stent-assisted coil embolization has also been reported ${ }^{8}$.

Endovascular stent-graft treatment is less invasive, and the technical success rate of this therapy is approximately $100 \%{ }^{2}$. Recently, some mid-term and long-term results have been reported in this field ${ }^{2,7,9}$. Hilfiker et al. reported primary patency and secondary patency rates of $89 \%$ and $100 \%$, respectively, in nine patients after a mean followup of 29 months (range 2-66 months) ${ }^{7}$. One patient experienced a stenosis at 12 months, and the patient was treated by angioplasty. Another patient had a thrombotic occlusion of stent graft because of a kink 2 months after the first treatment, and the patient was successfully treated by thrombolysis and a new stent placement ${ }^{7}$. Du Toit et al. reported long-term results of stent graft treatment of subclavian artery injuries in 25 patients with a mean follow-up duration of 49 months (range 5-104 months) ${ }^{9}$. Among them, five patients showed significant stenosis $(>50 \%)$, and three patients showed stent graft oc- 
clusion. All stenosis lesions were treated by angioplasty and were found to be patent during the follow-up. All occlusive lesions were treated conservatively, with no requirement for open surgery to improve symptoms or to achieve limb salvage during the long-term follow-up ${ }^{9}$. Other published series reported a few cases of stent-graft thrombosis, stenosis, and occlusion observed as complications, but these cases improved by angioplasty or were treated conservatively because the patients were asymptomatic ${ }^{4}$. In our case, mild stenosis occurred due to hyperplasia 8 months after the stent-graft implantation. The stenotic lesion was treated with a second angioplasty, and there was no limb ischemia.

No vascular stent graft is approved for use in this area by the Ministry of Health and Welfare in Japan. After the permission of the local ethical board, we decided to use the biliary Niti-S ComVi stent graft with PTFE cover.

In conclusion, we successfully treated a left subclavian artery pseudoaneurysm due to chopstick injury by stent graft. Mild stenosis occurred 8 months after the procedure, but the stenotic lesion was treated with a second angioplasty. Stent-graft treatment offers an attractive alternative to open surgery for the management of subclavian artery aneurysm due to its safety and efficacy.

Conflict of Interest: None of the authors have a conflict of interest to declare. This research did not receive any specific funding.

\section{References}

1. Cury M, Greenberg RK, Morales JP, Mohabbat W, Her- nandez AV: Supra-aortic vessels aneurysms: diagnosis and prompt intervention. J Vasc Surg 2009; 49: 4-10.

2. du Toit DF, Leith JG, Strauss DC, Blaszczyk M, Odendaal Jde V, Warren BL: Endovascular management of traumatic cervicothoracic arteriovenous fistula. $\mathrm{Br} \mathrm{J}$ Surg 2003; 90: 1516-1521.

3. Park SH, Cho KH, Shin YS, Kim SH, Ahn YH, Cho KG, Yoon SH: Penetrating craniofacial injuries in children with wooden and metal chopsticks. Pediatr Neurosurg 2006; 42: 138-146.

4. Assali AR, Sdringola S, Moustapha A, Rihner M, Denktas AE, Lefkowitz MA, Campbell M, Smalling RW: Endovascular repair of traumatic pseudoaneurysm by uncovered self-expandable stenting with or without transstent coiling of the aneurysm cavity. Catheter Cardiovasc Interv 2001; 53: 253-258.

5. Schönholz CJ, Uflacker R, De Gregorio MA, Parodi JC: Stent-graft treatment of trauma to the supra-aortic arteries. A review. J Cardiovasuc Surg (Torino) 2007; 48: 537549.

6. Becker GJ, Benenati JF, Zemel G, Sallee DS, Suarez CA, Roeren TK, Katzen BT: Percutaneous placement of a balloon-expandable intraluminal graft for life-threatening subclavian arterial hemorrhage. J Vasc Interv Radiol 1991; 2: 225-229.

7. Hilfiker PR, Razavi MK, Kee ST, Sze DY, Semba CP, Dake MD: Stent-graft therapy for subclavian artery aneurysms and fistulas: single-center mid-term results. J Vasc Interv Radiol 2000; 11: 578-584.

8. Sugawara T, Nakashima $K$, Yonenaga $K$, Watanabe $T$, Koyanagi T, Ito K, Kobori A, Baba Y: Successful stentassisted coil embolization for pseudoaneurysm of the left subclavian artery - a case report. Jpn J Intervent Radiol 2010; 25: 76-80.

9. du Toit DF, Lambrechts AV, Stark H, Warren BL: Longterm results of stent graft treatment of subclavian artery injuries: management of choice for stable patients? J Vasc Surg 2008; 47: 739-743.

(Received, April 27, 2016)

(Accepted, October 10, 2016) 